\title{
Genetic variation implicates plasma angiopoietin-2 in the development of acute kidney injury sub-phenotypes
}

Pavan K. Bhatraju ${ }^{1,2^{*}} \mathbb{D}$, Max Cohen ${ }^{1}$, Ryan J. Nagao ${ }^{3,4}$, Eric D. Morrell ${ }^{1}$, Susanna Kosamo ${ }^{1}$, Xin-Ya Chai ${ }^{1}$, Robin Nance ${ }^{5}$, Victoria Dmyterko ${ }^{1}$, Joseph Delaney ${ }^{5}$, Jason D. Christie ${ }^{6}$, Kathleen D. Liu', Carmen Mikacenic ${ }^{1}$, Sina A. Gharib ${ }^{1}$, W. Conrad Liles ${ }^{8}$, Ying Zheng ${ }^{3,4}$, David C. Christiani ${ }^{9,10}$, Jonathan Himmelfarb ${ }^{2}$ and Mark M. Wurfel ${ }^{1,2}$

\begin{abstract}
Background: We previously identified two acute kidney injury (AKI) sub-phenotypes (AKI-SP1 and AKI-SP2) with different risk of poor clinical outcomes and response to vasopressor therapy. Plasma biomarkers of endothelial dysfunction (tumor necrosis factor receptor-1, angiopoietin-1 and 2) differentiated the AKI sub-phenotypes. However, it is unknown whether these biomarkers are simply markers or causal mediators in the development of AKI sub-phenotypes.

Methods: We tested for associations between single-nucleotide polymorphisms within the Angiopoietin-1, Angiopoietin-2, and Tumor Necrosis Factor Receptor 1A genes and AKI- SP2 in 421 critically ill subjects of European ancestry. Top performing single-nucleotide polymorphisms (FDR <0.05) were tested for cis-biomarker expression and whether genetic risk for AKI-SP2 is mediated through circulating biomarkers. We also completed in vitro studies using human kidney microvascular endothelial cells. Finally, we calculated the renal clearance of plasma biomarkers using 20 different timed urine collections.

Results: A genetic variant, rs2920656C > T, near ANGPT2 was associated with reduced risk of AKI-SP2 (odds ratio, $0.45 ; 95 \% \mathrm{Cl}, 0.31-0.66$; adjusted FDR $=0.003)$ and decreased plasma angiopoietin-2 ( $p=0.002)$. Causal inference analysis showed that for each minor allele (T) the risk of developing AKI-SP2 decreases by $16 \%$. Plasma angiopoietin-2 mediated $41.5 \%$ of the rs 2920656 related risk for AKI-SP2. Human kidney microvascular endothelial cells carrying the T allele of rs2920656 produced numerically lower levels of angiopoietin-2 although this was not statistically significant $(p=0.07)$. Finally, analyses demonstrated that angiopoietin- 2 is minimally renally cleared in critically ill subjects.
\end{abstract}

Conclusion: Genetic mediation analysis provides supportive evidence that angiopoietin-2 plays a causal role in risk for AKI-SP2.

Keywords: Acute kidney injury, Genetics, Endothelium

\footnotetext{
* Correspondence: Bhatraju@uw.edu

'Division of Pulmonary, Critical Care and Sleep Medicine, Department of Medicine, University of Washington, 325 9th Avenue, Seattle, WA 98104, USA ${ }^{2}$ Kidney Research Institute, Division of Nephrology, Department of Medicine, University of Washington, Seattle, USA

Full list of author information is available at the end of the article
}

(C) The Author(s). 2020 Open Access This article is licensed under a Creative Commons Attribution 4.0 International License, which permits use, sharing, adaptation, distribution and reproduction in any medium or format, as long as you give appropriate credit to the original author(s) and the source, provide a link to the Creative Commons licence, and indicate if changes were made. The images or other third party material in this article are included in the article's Creative Commons licence, unless indicated otherwise in a credit line to the material. If material is not included in the article's Creative Commons licence and your intended use is not permitted by statutory regulation or exceeds the permitted use, you will need to obtain permission directly from the copyright holder. To view a copy of this licence, visit http://creativecommons.org/licenses/by/4.0/. The Creative Commons Public Domain Dedication waiver (http://creativecommons.org/publicdomain/zero/1.0/) applies to the data made available in this article, unless otherwise stated in a credit line to the data. 


\section{Background}

Acute kidney injury (AKI) affects $40-60 \%$ of patients admitted to the intensive care unit (ICU) [1] and contributes to poor short- and long-term outcomes [2-4]. Genetic studies to date have focused on associations between genetic variants and the risk for AKI comparing cases (AKI) to controls (no AKI) [5, 6]. However, this framework may be limited because cases of AKI are highly heterogeneous with different precipitants and biological profiles [7]. Combining such AKI patients to maximize sample size may result in dilution of genetic statistical signals that might only be present in one pathophysiologically distinct subset of the AKI population. Another limitation is that AKI in critically ill populations is often a complication of serious insult, such as sepsis, surgery, shock, pneumonia, and trauma. The use of controls without the development of AKI can be problematic. Controls carrying a high-risk genetic variant might not develop AKI if they do not also experience a similar acute insult as cases, and thus would be classified as non-cases, attenuating any potential association signal [8]. The use of biologically distinct AKI subphenotypes in genetic association studies overcomes prior limitations in phenotyping AKI by specifically focusing on the AKI population and by comparing two biologically distinct sub-phenotypes [9].

We recently identified two AKI sub-phenotypes (AKISP1 and AKI-SP2) applying latent class analysis methodology to a panel of 29 clinical and biomarker variables in two independent critically ill AKI populations [10]. Notably, AKI-SP2 was associated with worse hospital outcomes (e.g. mortality, new dialysis and 7-day renal non-recovery) compared to AKI-SP1. We next identified these AKI subphenotypes in a previously completed multi-center randomized control trial, Vasopressin versus Norepinephrine Infusion in Patients with Septic Shock (VASST) [11]. The VASST trial studied whether the choice of vasopressor therapy improved mortality in subjects with septic shock. While the AKI population in the clinical trial had no difference in mortality to vasopressor therapy, AKI-SP1 had a mortality benefit with vasopressin compared to AKI-SP2 having no mortality difference. To our knowledge, this is the first example of identifying treatment responsive AKI sub-groups in the critically ill.

Notably, no single variable was statistically better than the other variables to identify AKI-SP2 (Table S1). In contrast, a three-variable model, using plasma angiopoietin-2 (ANG-2), angiopoietin-1 (ANG-1), and soluble tumor necrosis factor receptor-1 (sTNFR-1), had the optimal predictive performance to differentiate AKI sub-phenotypes (C-statistic 0.93). Lower ANG-2, lower sTNFR-1 and higher ANG-1 were associated with lower risk of AKI-SP2. Studies in animal models of AKI have shown that these plasma biomarkers are involved in the pathophysiology and severity of AKI [12-16]. However, it is unknown whether these plasma biomarkers play a causal role in the development of clinical AKI subphenotypes. The identification of causal markers could inform targets for drug development to prevent or treat the development of AKI in the critically ill and could assist in patient risk-stratification.

Genetic mediation analysis is one of several causal inference approaches that can identify the potential mechanism by which an independent variable (e.g., genetic variant) affects the outcome (e.g., AKI sub-phenotypes) via an explanatory mediator (e.g., biomarker of endothelial dysfunction). This approach has been widely applied in clinical data to understand causal mechanisms of disease [17-19]. We hypothesized that cis-quantitative trait loci (QTLs) in the ANGPT1, ANGPT2, and TNFRSF1A genes influence the development of AKI sub-phenotypes by regulating circulating levels of their respective biomarkers (ANG-1, ANG-2 or sTNFR-1).

\section{Methods \\ Study populations}

We previously reported the identification of AKI subphenotypes using a prospectively collected ICU cohort: identification of Single Nucleotide Polymorphisms (SNPs) Predisposing to Altered Acute Lung Injury Risk (iSPAAR) $[10,20]$. The iSPAAR population is a genomewide case-control study of risk of acute respiratory distress syndrome (ARDS) that included patients with and without ARDS. The iSPAAR population included subjects from previously completed randomized control trials and from a prospectively enrolled ICU cohort. Details of the study design for each population enrolled in iSPAAR have been previously described; Albuterol for the Treatment of Acute Lung Injury (ALTA) [21], Fluid and Catheter Treatment Trial (FACTT) [22], Enteral omega-3 fatty acid, gamma-linolenic acid, and antioxidant supplementation in acute lung injury (OMEGA) [23] and Molecular Epidemiology of Acute Respiratory Distress (MEA) at the Massachusetts General Hospital [24]. Within iSPAAR, study enrollment occurred $48 \mathrm{~h}$ after ICU admission. At study enrollment DNA and plasma were collected for genotyping and biomarker analysis followed by AKI ascertainment. AKI was defined as an increase in serum creatinine $(\mathrm{SCr})$ of $\geq 0.3 \mathrm{mg} / \mathrm{dl}$ or $50 \%$ from "baseline" SCr. The baseline $\mathrm{SCr}$ was defined as the lowest value prior to study enrollment [25-27]. AKI was also defined using a modified urine output criteria (daily output instead of every $6 \mathrm{~h}$ ).

To determine renal clearance of plasma biomarkers, we enrolled a prospective cohort in the Harborview medical and surgical intensive care units, Critical Illness AKI Cohort (CIA). Subjects were eligible for enrollment if they met 2 of 4 systemic inflammatory response 
syndrome criteria, had a clinically-suspected infection, and had an indwelling urinary catheter in place. A timed urine collection was completed that lasted at least $2-4 \mathrm{~h}$ and EDTA plasma samples were collected at the beginning and the end of the timed urine collection. Clearance was calculated using the formula Clearance $(X)=U(X)$ * $V / P(X)$, where $U(X)$ represents the urine concentration of solute $X, V$ indicates the urine volume over the 2-4-h collection period, and $P(X)$ represents the average plasma concentrations of solute $X$ from the initial and final blood collections.

\section{Genotyping strategy}

Genotyping was performed in 421 patients of European ancestry using the Illumina 660 platform (Illumina, San Diego, CA). Genotyped data was quality controlled using a sample call rate filter $>0.97$, minor allele frequency (MAF) $>0.01$ and SNP call rate $>0.95$. After quality control, 238 SNPs \pm 50 kilobases of ANGPT1, ANGPT2 and TNFRSF1A were found. After linkage disequilibrium (LD) pruning of a $\mathrm{r}^{2}$ of $0.8,48$ SNPs were removed leading to a total of 190 SNPs used in association tests. Imputation was conducted using the 1000 Genomes Project reference panel using IMPUTE2 v2.3.0 [28, 29].

\section{Plasma protein assessment}

Plasma and urinary biomarkers were measured using electrochemiluminescent immunoassays (Meso Scale Discovery (MSD), Rockville, MD), as previously described [10]. The blood was collected in EDTA-treated sterile tubes, urine was collected in sterile containers and both were centrifuged immediately. Plasma and urine was then aliquoted and frozen at $-80^{\circ} \mathrm{C}$. The samples were stored for different durations but they were thawed in a single batch and only once for running the biomarker measurements for this study. All biomarker measurements were performed in duplicates at Harborview Pulmonary Research Laboratories.

\section{In Silico analyses}

To test SNPs for expression of QTL effects, we queried the Genotype-Tissue Expression (GTEx) Portal [30].

\section{Cell culture}

Human kidney microvascular endothelial cells (HKME Cs) were purified from fetal kidneys after voluntary pregnancy interruptions between 100 and 135 days postconception. Informed consents for the use of fetal tissues were obtained from patients. We then randomly chose 9 different donor HKMECs, thawed and plated half a million cells in T25 flasks coated with $0.2 \%$ gelatin and maintained in EBM-2 basal medium containing 1\% antibiotic-antimycotic (Life Technologies), 10\% FBS, $100 \mu \mathrm{g} / \mathrm{mL}$ ECGS, $50 \mu \mathrm{g} / \mathrm{mL}$ Heparin, and $20 \mathrm{ng} / \mathrm{mL}$
VEGF (R\&D), for $48 \mathrm{~h}$ till confluency. At $48 \mathrm{~h}$, we purified genomic DNA from the HKMECs and cell supernatants were collected. We successfully genotyped cells from 8 donors.

\section{Statistical analysis}

Patient demographic variables are reported as either mean $+/$-standard deviation or as median and quartiles. First, we used logistic regression to test for an association between the 190 SNPs and the development of AKI-SP2 compared to AKI-SP1 using an additive genetic model (Golden Helix, MT). Our model was adjusted for the following covariates: age, sex, sepsis and first five principal components. The Eigenstrat method $\mathrm{v} 4.2$ was used to calculate the principal components and the top five were included as covariates. Odds ratios (ORs) are reported with 95\% confidence intervals. For the analysis between genetic variants and AKI subphenotypes, we corrected for multiple comparisons by using a Benjamini-Hochberg false discovery rate (FDR) threshold $<0.10$, which estimates that less than $10 \%$ of the associations with an FDR value at or below this level are false positives [31]. In a sensitivity analysis, an imputed genotype was used to identify additional SNPs associated with AKI-SP2.

Second, we used linear regression adjusting for age, sex and sepsis to determine associations between top performing SNPs and $\log _{2}$ transformed biomarker concentrations. Third, we completed causal inference analysis to test the association between genetic variants and AKI-SP2 and the potential mediation of the association by plasma biomarker concentrations. The mediation analysis was performed using the nonlinear implementation of structural equation modeling implemented in the mediation package for STATA [32, 33]. Additional details of the causal inference analysis are provided in the online supplement. Fourth, we determined associations between genetic variants and AKI severity, measured by maximum serum creatinine, via logistic regression. Additional details of materials and methods are provided in the online supplement. In the analysis, we evaluated 190 SNPs and used a conservative p-value of $0.05 / 190=$ $2.6 \times 10^{-4}$. Given that approximately $40 \%$ of ICU patients develop AKI, and an expected control (AKISP1) to case (AKI-SP2) ratio of 1.5, an expected sample size of 421 , and a MAF of at least 0.30 , we will have $81 \%$ power to detect a relative risk of 1.5 or greater [34]. Analyses were completed using STATA (Version 15) and Goldenhelix (Version 4.0). All studies were approved by the Human Subjects Division at the University of Washington. Written informed consent was obtained from all subjects enrolled. 


\section{Results}

\section{Characteristics of populations}

Of the 425 patients from the validation cohort in our previous work, 421 had genotyping data available. Demographics and baseline clinical characteristics are described in Table 1. All subjects were of European ancestry. A total of 267 (63\%) were classified as AKI-SP1 and 154 (37\%) as AKI-SP2. Subjects who developed AKI-SP2 had higher illness severity on presentation (mean acute physiology and chronic health evaluation (APACHE) III scores, $111 \pm 26$ vs $74 \pm 24)$, were more likely to have sepsis $(84 \%$ vs $66 \%)$ and were more likely to be treated with vasopressors (79\% vs $42 \%$ ) compared to AKI-SP1.

\section{Genetic Variation Near ANGPT2, rs2920656, is Associated with AKI-SP2}

Of the 190 SNPs \pm 50 kilobases of the genes, 72 were near ANGPT1, 100 were near ANGPT2 and 18 near TNFRSF1A gene. We identified one SNP meeting an FDR $<0.05$ that was associated with AKI-SP2 compared to AKI-SP1 (Table 2 and Fig. 1). No significant associations were observed with SNPs in or near ANGPT1 or TNFRSF1A (Table S2 and S3).

The SNP demonstrating the strongest association with risk for AKI-SP2 was rs2920656 (OR, 0.45; 95\% CI, $\left.0.31-0.66 ; p<1.4 \times 10^{-5} ; \quad \mathrm{FDR}=0.003\right)$. This intronic $\mathrm{SNP}$ is $\approx 30 \mathrm{~kb}$ downstream to the $3^{\prime}$ position of ANGP $T 2$ and explained approximately $3 \%$ of the variance in

Table 1 Demographic and clinical data

\begin{tabular}{|c|c|c|c|}
\hline Participant Characteristics & AKI-SP1 $(\boldsymbol{N}=267)$ & AKI-SP2 $(\boldsymbol{N}=154)$ & $p$-value \\
\hline \multicolumn{4}{|l|}{ Baseline Demographics } \\
\hline Age (year) & $57 \pm 18$ & $56 \pm 17$ & 0.84 \\
\hline Male (\%) & $164(61)$ & $104(68)$ & 0.19 \\
\hline Body Mass Index (kg/m²) & $29 \pm 8$ & $29 \pm 8$ & 0.97 \\
\hline \multicolumn{4}{|l|}{ Race (\%) } \\
\hline Caucasian & $267(100)$ & $154(100)$ & 1.00 \\
\hline \multicolumn{4}{|l|}{ Co-Morbidities } \\
\hline Diabetes Mellitus & $72(27)$ & $40(26)$ & 0.69 \\
\hline Cirrhosis & $6(2)$ & $14(9)$ & $<0.01$ \\
\hline \multicolumn{4}{|l|}{ ICU Events ${ }^{a}$} \\
\hline APACHE III Scores & $74 \pm 24$ & $111 \pm 26$ & $<0.01$ \\
\hline Sepsis - 3 & $178(67)$ & $132(86)$ & $<0.01$ \\
\hline Vasopressors & $113(42)$ & $124(81)$ & $<0.01$ \\
\hline $24 \mathrm{~h}$ urine output (ml) & $1680(1140-2665)$ & $1199(563-2050)$ & $<0.01$ \\
\hline \multicolumn{4}{|l|}{ ICU Laboratory Values $^{\mathrm{a}}$} \\
\hline Maximum White Blood Cell Count (10*9) & $16 \pm 8$ & $17 \pm 13$ & 0.25 \\
\hline Low Hematocrit (\%) & $30 \pm 6$ & $31 \pm 6$ & 0.74 \\
\hline Low Sodium (mEq/L) & $137 \pm 6$ & $135 \pm 5$ & $<0.01$ \\
\hline Low Albumin (g/dL) & $2.4 \pm 0.6$ & $2.2 \pm 0.7$ & $<0.01$ \\
\hline Low Platelets $\left(10^{9} / \mathrm{L}\right)$ & $184 \pm 101$ & $85 \pm 75$ & $<0.01$ \\
\hline Low Sodium Bicarbonate (mEq/L) & $22 \pm 5$ & $17 \pm 5$ & $<0.01$ \\
\hline \multicolumn{4}{|l|}{ Biomarker Concentrations (pg/ml) } \\
\hline Angiopoietin-2 & $23,458(12,208-38,707)$ & $74,972(48,294-128,421)$ & $<0.01$ \\
\hline Angiopoietin-1 & $2361(1090-5102)$ & 778 (398-1998) & $<0.01$ \\
\hline Soluble Tumor Necrosis Factor Receptor-1 & $10,581(6828-15,742)$ & $25,815(16,084-36,211)$ & $<0.01$ \\
\hline \multicolumn{4}{|l|}{ Outcomes } \\
\hline Length of ICU stay, d & $7.5 \pm 7.3$ & $8.3 \pm 8.7$ & $<0.01$ \\
\hline Maximum 7 day Serum Creatinine (mg/dL) & $1.6(1.2-2.3)$ & $2.7(1.8-4.3)$ & $<0.01$ \\
\hline 28-day mortality & $36(14)$ & $57(37)$ & $<0.01$ \\
\hline
\end{tabular}

Data shown as mean \pm standard deviation, $\mathrm{n}(\%)$, median (interquartile range), as appropriate. ${ }^{\mathrm{a}} \mathrm{All}$ ICU Events and ICU laboratory values are the maximum or minimum value at the time of study enrollment 
Table 2 SNPs Most Associated with AKI-SP2

\begin{tabular}{llllllll}
\hline SNP & Associated Gene & $\begin{array}{l}\text { Chromosome: } \\
\text { Coordinate }\end{array}$ & Function & $\begin{array}{l}\text { Minor Allele } \\
\text { Frequency } \\
(1000 \text { Genomes })\end{array}$ & $\begin{array}{l}\text { Odds Ratio } \\
(95 \% \text { Cl) }\end{array}$ & P-value & FDR Corrected $\boldsymbol{P}$-value \\
\hline 1. rs2920656 & MCPH1 & Chr8:6329510 & Intron & 0.284 & $0.45(0.31-0.66)$ & $1.38 \times 10^{-5}$ & 0.0026 \\
2. rs2442473 & ANGPT2 MCPH1 & Chr8:6358293 & Intron & 0.082 & $0.40(0.22-0.74)$ & 0.001902 & 0.0723 \\
3. rs2920689 & MCPH1 & Chr8:6343499 & Intron & 0.310 & $0.59(0.42-0.82)$ & 0.001538 & 0.0731 \\
4. rs2959779 & MCPH1 & Chr8:6443380 & Intron & 0.055 & $0.27(0.11-0.67)$ & 0.001307 & 0.0828 \\
5. rs2440399 & MCPH1 & Chr8:6325975 & Intron & 0.207 & $0.52(0.34-0.78)$ & 0.000984 & 0.0935 \\
\hline
\end{tabular}

Definition of abbreviations: SNP single nucleotide polymorphism, Cl confidence interval, FDR False Discovery Rate

${ }^{a}$ Additive genetic model adjusted for age, gender, sepsis and 5 principal components

development of AKI-SP2 $\left(R^{2}\right)$. Because only a small number of subjects were homozygous rs2920656 $(n=$ 26), we also tested associations between rs2920656 and AKI-SP2 in a dominant genetic model, which gave consistent results (OR, 0.42; 95\% CI, 0.28-0.64; $p<1.4 \times$ $10^{-6}$ ). We also tested for additional, potentially stronger, associations within the ANGPT2 locus using imputed genotypes but did not find any associations stronger than that observed with rs2920656 (Table S4).

In a sensitivity analysis we tested whether the inclusion of critically ill patients without AKI would influence the genetic association. We grouped patients with no AKI and AKI-SP1 together and determined whether rs2920656 was still strongly associated with a decreased risk of AKI-SP2. In this analysis, 839 patients had either no AKI or AKI-SP1 and 154 had AKI-SP2. The risk of developing AKI-SP2 again was significantly reduced with having at least one $\mathrm{T}$ allele for rs2920656 (OR 0.31; 95\% CI, 0.19-0.52; $p<0.001$ ) (Table S5). In another sensitivity analysis, we tested if rs2920656 was associated with decreased risk of AKISP2 within each of the four different studies that were

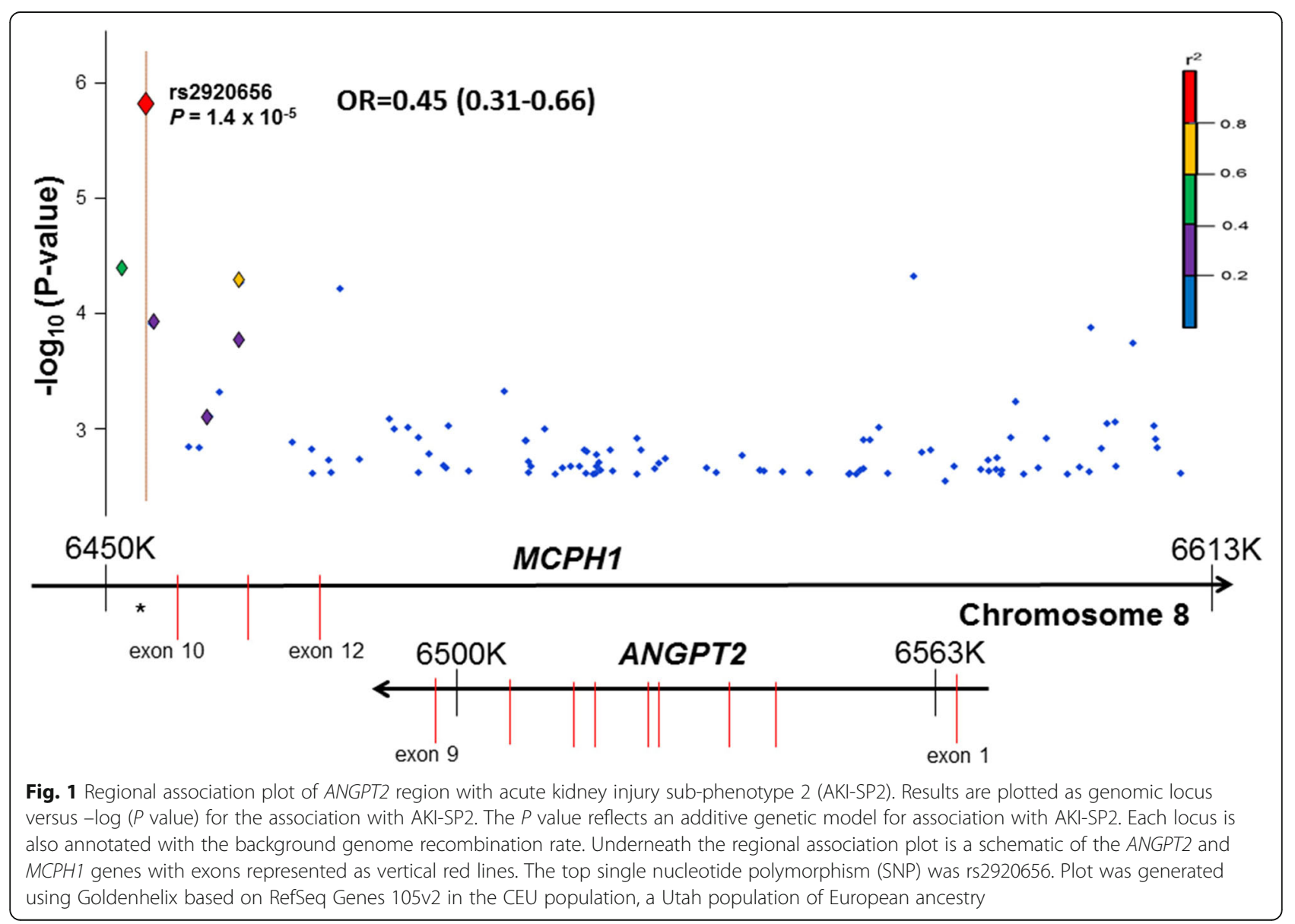


included in iSPAAR. Within each of the three randomized control trials (ALTA, FACTT and OMEGA) and the ICU prospective cohort (MEA), the point estimate was consistent with the minor allele of rs2920656 demonstrating a decreased risk for the development of AKI-SP2 (Table S6). Thus, rs2920656 was analyzed further to determine the association with plasma biomarker concentrations.

T allele of rs2920656 is associated with decreased plasma ANG-2

We next analyzed the association between rs2920656 and plasma ANG-2 concentrations. Adjusting for age, gender and sepsis each copy of the T allele of rs2920656 was associated with decreased $\log _{2}$ plasma ANG-2 concentrations $(\beta=-0.09 ; 95 \% \mathrm{CI}-0.15,-0.04 ; P=0.002)$. Subjects homozygous for the $C$ allele showed the highest concentrations of plasma ANG-2 $(40,683 \mathrm{pg} / \mathrm{ml}$; interquartile range (IQR) 19,374-73,205), while subjects homozygous for the $\mathrm{T}$ allele showed the lowest plasma ANG-2 concentrations (28,308 pg/ml (IQR 14,340-42, 944). In addition, of the 100 SNPs tested near the ANGP T2 gene region, rs29206565 was the most strongly associated with plasma ANG-2 concentrations (Fig. 2).
Mediation analysis suggests ANG-2 is causal in the development of AKI-SP2

We tested for evidence that the association between rs2920656 and risk for AKI-SP2 is mediated through plasma ANG-2 concentrations (Fig. 3). The total effect of rs2920656 on AKI-SP2 was $\beta_{\text {total }}=-0.16$ per allele (95\% CI $\left.-0.24,-0.10, p=1.0 \times 10^{-4}\right)$, which suggests that for each minor allele ( $\mathrm{T}$ allele) of the genetic variant the risk of developing AKI-SP2 decreases by $16 \%$. Causal mediation analysis detected a significant indirect effect for rs2920656 on AKI-SP2 that was mediated through plasma ANG-2 concentrations $\left(\beta_{\text {indirect }},-0.07\right.$ per allele; $95 \%$ CI $-0.11,-0.03 ; p=0.001)$, which means the proportion of effect between rs2920656 and AKI-SP2 that is mediated by ANG- 2 concentrations is $41.5 \%$.

\section{T allele of rs2920656 is associated with decreased AKI severity at 7 days}

Next, we tested the association of rs2920656 with traditional criteria for AKI severity, such as maximum serum creatinine and increase in serum creatinine within 7 days after study enrollment. In a dominant genetic model, the $\mathrm{T}$ allele of rs2920656 was associated with a decrease in serum creatinine of $-0.44 \mathrm{mg} / \mathrm{dL}(95 \% \mathrm{CI},-0.77$, $0.10), p=0.01$ ) after adjusting for age, gender, body mass

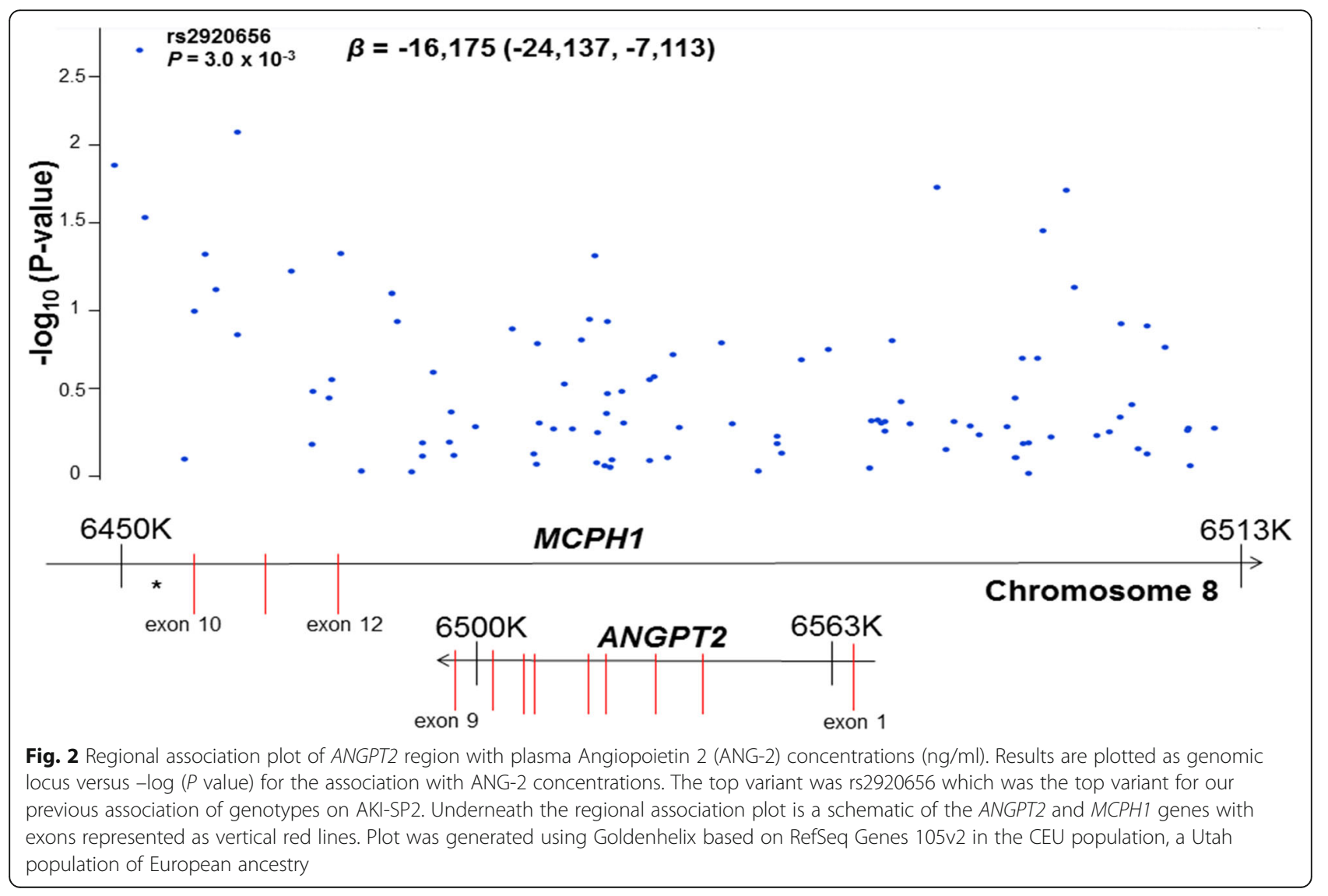




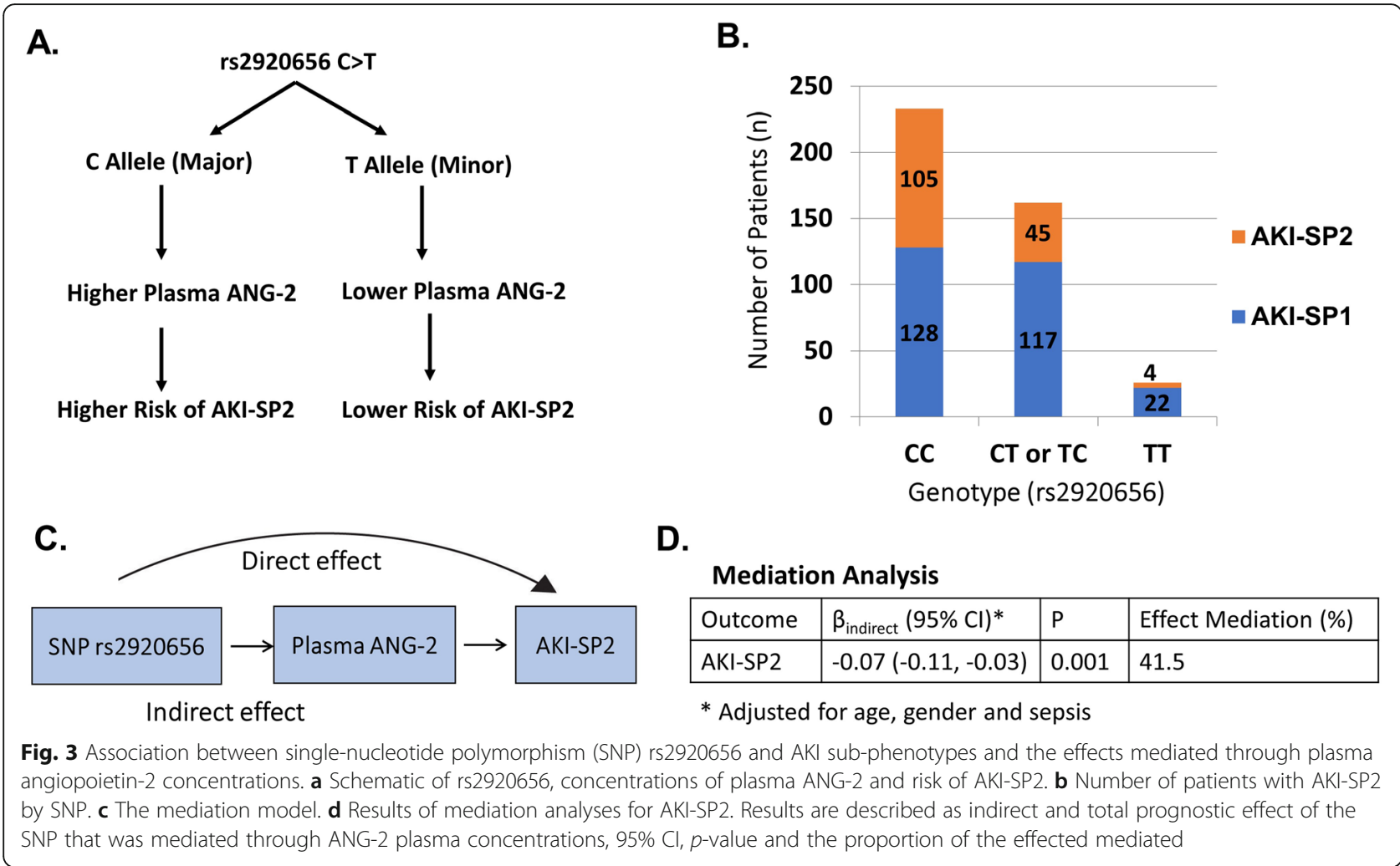

index and sepsis status. The minor allele of rs2920656 was also associated with a decrease in the rise in serum creatinine between baseline and day $7 \quad(\beta=-0.25,95 \%$ CI, $-0.46,-0.03 ; p=0.03$ ).

\section{T allele of rs2920656 is associated with lower ANG-2 in cell culture}

We conducted in vitro experiments and in silico analyses to determine the functional significance of rs2920656. Of 8 different human fetal kidney tissue samples, 2 were CC, 5 were CT and 1 was TT for rs2920656. In a dominant genetic model, ANG-2 concentrations were numerically greatest in endothelial cells from donors homozygous for the $\mathrm{C}$ allele and lower in carriers of the $\mathrm{T}$ allele, $p=0.07$ (Fig. 4). In the GTEx project database, rs2920656 was not associated with ANGPT2 gene expression. However, two other SNPs (rs41311412 and rs2515591) that are in moderate LD $\left(r^{2}=0.23\right.$ and $D^{\prime}=$ $0.86)$ with rs2920656 were associated with reduced ANGPT2 gene expression $\left(p=4.1 \times 10^{-5}\right)$ in tibial artery, a tissue which is highly enriched for endothelial cells (Table S7).

\section{Plasma ANG-2 is minimally cleared by the kidneys}

To determine whether differences in kidney function could influence plasma ANG-2 concentrations, we measured ANG-2 renal clearance in critically ill subjects with and without AKI. In 20 different timed urine sample collections with bookended plasma samples, the median serum creatinine was $0.86 \mathrm{mg} / \mathrm{dL}$ with an interquartile range (IQR) 0.69 to $1.45 \mathrm{mg} / \mathrm{dL}$. The median plasma ANG-2 concentration was $10,261 \mathrm{pg} / \mathrm{mL}$ (IQR 6210-19,115 pg/mL). In contrast, urinary ANG-2 concentrations were 50 -fold lower with a median of $206 \mathrm{pg} /$ $\mathrm{mL}$ (IQR $11-839 \mathrm{pg} / \mathrm{mL}$ ). The calculated renal clearance of ANG-2 was $<1 \mathrm{~mL} / \mathrm{min}$ for all timed urine collections, suggesting that plasma ANG-2 concentrations are not increased simply as a function of worsening AKI (Table 3).

\section{Discussion}

The search for susceptibility genes in AKI has been hindered by heterogeneity within the clinical disease phenotype. Using AKI sub-phenotypes, we have discovered that a genetic variant near the ANGPT2 gene, rs2920656, is protective against the development of AKISP2. Causal inference analysis suggests that plasma ANG-2 concentrations mediated $41.5 \%$ of the genetic association between rs2920656 and AKI-SP2 risk in subjects of European ancestry. Furthermore, in-vitro experiments demonstrated that the minor allele of rs2920656 may lead to lower ANG-2 protein release by human kidney endothelial cells. In addition, plasma ANG-2 is minimally renally cleared, suggesting that elevated plasma ANG-2 concentrations are unlikely to result from kidney injury. Overall, these findings provide 


\section{ANG-2 in Endothelial Cell Culture}

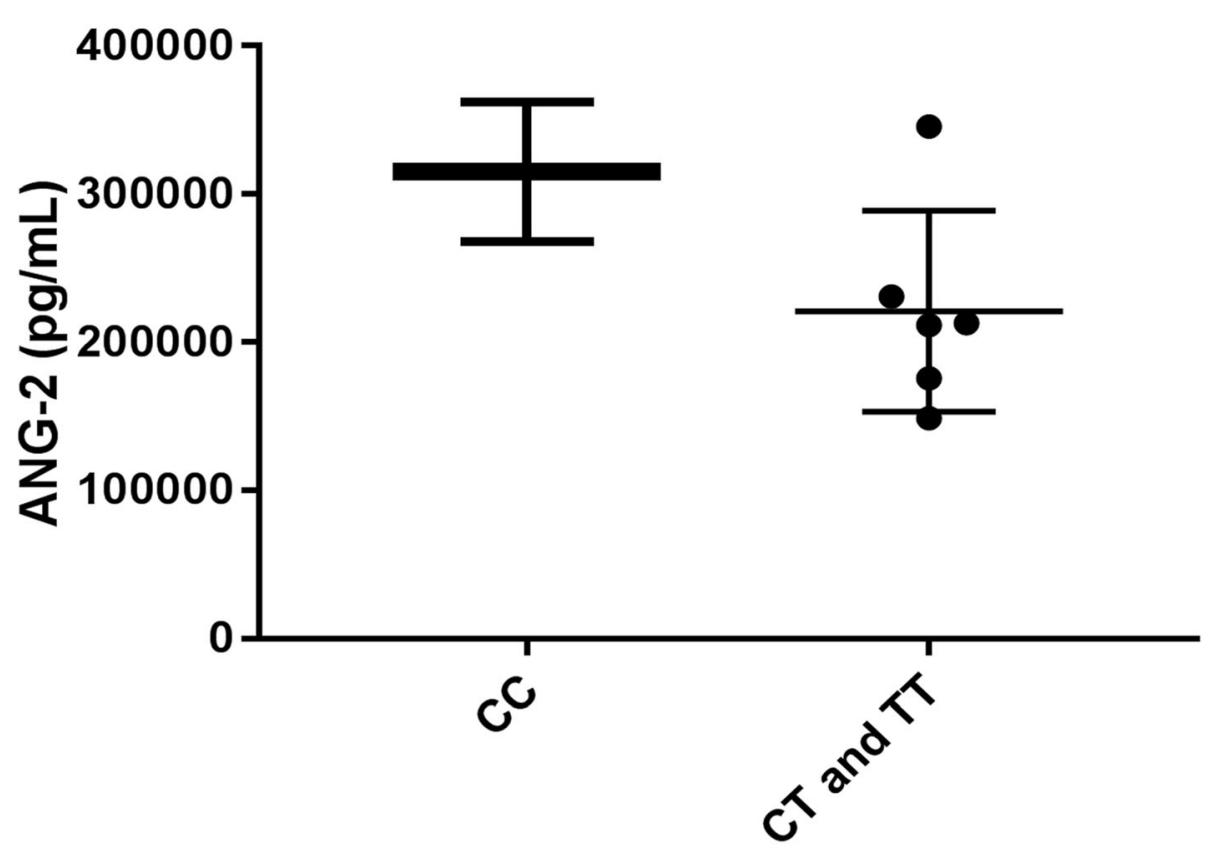

rs2920656 Genotype

Fig. 4 ANG-2 concentrations by rs2920656 genotype in human microvascular kidney endothelial cells (HKMECs). Of 8 human kidney samples, 2 were CC, 5 were CT and 1 was TT for rs2920656. The T allele of rs2920656 was associated with decreased ANG-2 concentrations. Student t-test was used combining the $C T$ and $T T$ genotypes and generated a $p$-value $=0.07$ for the difference between genotypes and ANG-2 concentrations. The mean ANG-2 concentrations are 315,000 pg/mL for CC, $220,900 \mathrm{pg} / \mathrm{mL}$ for CT and $T T$

evidence that plasma ANG-2 plays a mechanistic role in the host's response to critical illness leading to AKI-SP2. Efforts to target the Ang-Tie2 axis may limit AKI severity and resulting poor clinical outcomes [35].

The ANPT2 gene is $100 \%$ nested within the microcephalin (MCPH1) gene. Mutations in the MCPH1 gene have been associated with diseases of neurogenesis and renal cell carcinoma [36]. We have shown that rs2920656 is an intronic variant in $\mathrm{MCPH} 1$ that regulates plasma ANG-2 concentrations. Previous studies have identified genetic variants in MCPH1 [37] and ANGPT2 [38] that are associated with ANG-2 concentrations. It is also conceivable that rs2920656 influences $M C P H 1$ expression. However, to our knowledge no role for $\mathrm{MCPH1}$ in acute or chronic kidney disease or vascular injury has been described. In contrast, multiple pre-clinical and clinical studies have demonstrated the important role of plasma ANG-2 in the development of AKI [16, 39, 40].

Multiple reports have implicated plasma biomarkers of endothelial function in the pathophysiology of AKI [39, 41, 42]. ANG-1 and -2 are vascular endothelial growth factors that both bind to the endothelial tyrosine kinase receptor (Tie-2) but have context dependent activities
[43]. ANG-1 is released by pericytes and platelets and is an agonist for the Tie-2 receptor. ANG-1 is protective by stabilizing the endothelium and preventing microcirculatory capillary leakage, a hallmark of AKI [44]. In contrast, ANG-2 typically acts as an antagonist to the Tie-2 receptor and promotes endothelial permeability [45] and inflammation [46, 47]. The ANGPT2 gene encodes for circulating ANG-2, which is released from endothelial cells during an inflammatory stimulus. Animal studies have shown that inhibition of ANG-2 binding, augmenting ANG-1 concentrations [41, 44], or activation of Tie-2 [46] decreases endothelial leak and protects against AKI. Taken together these studies implicate a mechanistic role of the ANG-Tie2 axis in AKI. Here we demonstrate genomic regulation of plasma ANG-2 as another piece supporting the causative role of ANG-2 in the development of a severe form of AKI, AKI-SP2.

The strong association between plasma ANG-2 and development of AKI-SP2, raises the question of whether ANG-2 is similar to creatinine: filtered by the kidney and elevated levels are simply reflections of decreased renal filtration. To demonstrate that plasma ANG-2 
Table 3 ANG-2 Renal Clearance in Subjects with and without AKI

\begin{tabular}{|c|c|c|c|c|}
\hline $\begin{array}{l}\text { Timed Urine } \\
\text { Collection }\end{array}$ & $\begin{array}{l}\text { Average Serum } \\
\text { Creatinine (mg/dL) }\end{array}$ & $\begin{array}{l}\text { Average Plasma } \\
\text { ANG-2 (pg/mL) }\end{array}$ & $\begin{array}{l}\text { Urine ANG2 } \\
\text { (pg/mL) }\end{array}$ & $\begin{array}{l}\text { ANG-2 Renal } \\
\text { Clearance }(\mathrm{mL} / \mathrm{min})\end{array}$ \\
\hline 1 & 3.24 & $29,312.89$ & 4671.87 & 0.216 \\
\hline 2 & 3.52 & $27,136.51$ & 5915.63 & 0.353 \\
\hline 3 & 0.82 & 5061.54 & 10.00 & 0.002 \\
\hline 4 & 0.89 & 4782.57 & 553.81 & 0.161 \\
\hline 5 & 0.61 & 4435.69 & 169.09 & 0.064 \\
\hline 6 & 0.53 & 3867.01 & 10.00 & 0.006 \\
\hline 7 & 0.75 & $10,426.38$ & 11.80 & 0.004 \\
\hline 8 & 0.69 & 9699.06 & 14.58 & 0.002 \\
\hline 9 & 0.60 & 6593.72 & 10.00 & 0.004 \\
\hline 10 & 0.82 & 8350.90 & 190.68 & 0.010 \\
\hline 11 & 0.71 & $15,886.06$ & 220.09 & 0.003 \\
\hline 12 & 0.69 & $10,096.66$ & 243.74 & 0.018 \\
\hline 13 & 2.57 & $26,288.02$ & 2097.31 & 0.079 \\
\hline 14 & 2.24 & $24,769.31$ & 1967.59 & 0.165 \\
\hline 15 & 2.16 & $22,259.65$ & 1696.53 & 0.074 \\
\hline 16 & 0.99 & $10,839.17$ & 10.00 & 0.002 \\
\hline 17 & 0.44 & 2624.28 & 10.00 & 0.007 \\
\hline 18 & 1.20 & $18,067.95$ & 192.79 & 0.014 \\
\hline 19 & 1.14 & $13,683.26$ & 527.93 & 0.069 \\
\hline 20 & 1.13 & 8829.19 & 245.35 & 0.095 \\
\hline
\end{tabular}

Serum creatinine and plasma ANG-2 calculated based on the average measurement from the plasma sample collected at the beginning and at the end of the time urine collection. Clearance calculated using the formula Clearance $(X)=U(X) * V / P(X)$, where $U(X)$ represents the urine concentration of solute $X, V$ indicates the urine volume over the $2-4$-h collection period, and $P(X)$ represents the average plasma concentrations of solute $X$ from the initial and final blood collection

concentrations are not simply a marker but causal in the development of severe AKI, we provide two lines of evidence. First, using genetic causal inference analysis, we have shown that genetic variation near the ANGPT2 gene is associated with ANG-2 plasma concentrations and the development of AKI-SP2. Second, unlike creatinine (113 Da), ANG-2 (57,000 Da) is a large molecule that is unlikely to be regularly filtered at the glomerulus. In a critically ill population, we have demonstrated minimal renal clearance of ANG-2. Thus, elevations in plasma ANG-2 concentrations are unlikely to be due to differences in renal filtration and, instead, may be involved in the pathophysiology of AKI in critically ill patients.

It is important to note that individual genetic variants likely have small overall effects on disease development because AKI is likely a polygenic disease. The strength of this analysis is identification of a genetic variant that supports ANG-2 as causal in the development of AKI. Even variants with modest effect sizes provide opportunities for the investigation of potential novel causal pathways using genetic medication analysis. For example, cardiovascular disease, similar to AKI, is a polygenic trait with many genetic variants each explaining a small proportion of the risk. Regardless, three SNPs that explained only 0.4 to $2 \%$ of the variance in c-reactive protein levels allowed the determination that c-reactive protein was not causal in the development of ischemic vascular disease [48], and these findings were confirmed in subsequent studies [49].

Our work has several strengths. First, we used AKI sub-phenotypes to leverage precision in the phenotype definition and to maximize sample size to discover genetic variants. Second, causal inference analysis suggests that $41.5 \%$ of rs2920656-associated risk for developing AKI-SP2 is explained by plasma ANG-2 levels. This provides clinical evidence, to build on work from animal studies, that modulation of plasma ANG-2 concentrations may improve outcomes in critical illness associated AKI. Third, to account for potential residual confounding and to link Ang-2 production specifically to kidney endothelial cells, we completed in-vitro experiments using HKMECs. Fourth, using a unique ICU cohort with timed urine collection samples and before and after plasma samples, we were able to demonstrate that minimal amounts of plasma ANG-2 is filtered by the kidney. Thus, the strong association of ANG-2 with kidney specific outcomes is likely not confounded by issues of reverse causation. 
Our study has limitations. Our sample size was relatively small. However, our well-defined quantitative trait (AKI-SP2) allowed us to identify a genetic association with the limited number of critically ill patients with AKI. Second, analyses were limited to patients of European ancestry in order to reduce genetic admixture, maximize power and because of differences in allelic frequencies among ethnic backgrounds. Future work is warranted to study alternative ethnic populations to determine if similar genomic variation influences plasma ANG-2 concentrations. Third, while there was a trend in lower ANG-2 measurements in PTECs with the minor allele, the results were not statistically significant. However, the human samples are difficult to obtain and even with a small sample size we saw a consistent direction. Fourth, due to the uniqueness of this dataset we were unable to find a similar patient population to replicate our findings. Our dataset included well-phenotyped patients with AKI, with genomic, plasma and clinical outcome data. However, within the four individual populations included in iSPAAR there was a consistent direction in effect between rs2920656 and development of AKI-SP2. Future work is warranted to understand the influence of rs2920656 on sub-phenotype development and AKI specific clinical outcomes.

\section{Conclusion}

In summary, we identified a genetic variant near the ANGPT2 gene that is associated with plasma ANG-2 concentrations and the development of AKI-SP2 among a critically ill population. We also tested this association through studies completed in HKMECs. Our findings suggest that plasma ANG-2 plays a causal role in the development of AKI-SP2 and believe efforts to target the Ang-Tie2 axis may prevent the development of poor clinical outcomes.

\section{Supplementary information}

Supplementary information accompanies this paper at https://doi.org/10. 1186/s12882-020-01935-1.

Additional file 1: Supplement Data File. Includes supplement tables referenced in the manuscript.

\section{Abbreviations}

AKI: Acute kidney injury; AKI-SP1: Acute kidney injury sub-phenotype 1; AKISP2: Acute kidney injury sub-phenotype 2; ANG-1: Angiopoietin 1; ANG2: Angiopoietin 2; ANGPT1: Angiopoietin-1 gene; ANGPT2: Angiopoietin-2 gene; APACHE III: Acute Physiology Age Chronic Health Evaluation; Cl: Confidence Interval; ICU: Intensive Care Unit; KDIGO: Kidney Disease Improving Global Outcomes; MAF: Minor allele frequency; SNP: Singlenucleotide polymorphism; sTNFR-1: Soluble Tumor Necrosis Factor Receptor - 1; TNFRSF1A: Tumor necrosis factor receptor surface-1A

\section{Acknowledgements}

The authors acknowledge the supportive role of all support staff and participating patients in the study.

\section{Authors' contributions}

Study concept and design: $\mathrm{PKB}, \mathrm{JH}, \mathrm{MMW}$; Acquisition, analysis, or interpretation of data: PKB, MC, RJN, EDM, SK, XYC, RN, VD, JD, JDC, KDL, CM, SAG, WCL, YZ, $\mathrm{DCC}, \mathrm{JH}, \mathrm{MMW}$; Drafting of the manuscript: PKB; Critical revision of the manuscript for important intellectual content: PKB, MC, RJN, EDM, SK, XYC, RN, VD, JD, JDC, KDL, CM, SAG, WCL, YZ, DCC, JH, MMW; Statistical analysis: PKB, RN, JD; Study supervision: JH, WCL, MMW. All authors have read and approved this manuscript.

\section{Funding}

This work was supported by grants from the National Heart, Lung, and Blood Institute R01HL060710, the National Institute of Diabetes, Digestive and Kidney Diseases K23DK116967, the National Institutes of Health Grants UH2/ UH3 TR000504 and an unrestricted gift to the Kidney Research Institute from the Northwest Kidney Centers. The funding sources had no role in design and conduct of the study; collection, management, analysis, and interpretation of the data; and preparation, review, or approval of the manuscript.

\section{Availability of data and materials}

The datasets generated during and/or analyzed during the current study are available in the dbGAP genotypes and phenotypes repository, accession number: phs000631.v1.p1 and web link is https://www.ncbi.nlm.nih.gov/ projects/gap/cgi-bin/study.cgi?study_id=phs000631.v1.p1

\section{Ethics approval and consent to participate}

The University of Washington Institutional Review Board approved this study. All patients provided necessary written informed consent to participate in this study.

\section{Consent for publication}

No individual personal data is included in the study. All patients provided necessary consent to participate in this study, including consent to participate in the study where appropriate.

\section{Competing interests}

None of the authors have competing financial interests.

\section{Author details}

'Division of Pulmonary, Critical Care and Sleep Medicine, Department of Medicine, University of Washington, 325 9th Avenue, Seattle, WA 98104, USA. ${ }^{2}$ Kidney Research Institute, Division of Nephrology, Department of Medicine, University of Washington, Seattle, USA. ${ }^{3}$ Department of Bioengineering, University of Washington and Center for Cardiovascular Biology, Seattle, USA. ${ }^{4}$ Institute of Stem Cell and Regenerative Medicine, University of Washington, Seattle, USA. ${ }^{5}$ Department of Epidemiology, University of Washington,

Seattle, USA. ${ }^{6}$ Division of Pulmonary, Allergy, and Critical Care and Center for Clinical Epidemiology and Biostatistics, Department of Medicine, University of Pennsylvania, Philadelphia, USA. ${ }^{7}$ Divisions of Nephrology and Critical Care Medicine, University of California San Francisco, San Francisco, USA.

${ }^{8}$ Department of Medicine, University of Washington, Seattle, USA.

${ }^{9}$ Departments of Environmental Health and Epidemiology, Harvard TH Chan School of Public Health, Harvard University and Pulmonary and Critical Care Division, Cambridge, USA. ${ }^{10}$ Department of Medicine, MA General Hospital/ Harvard Medical School, Boston, USA.

Received: 5 July 2019 Accepted: 7 July 2020

Published online: 17 July 2020

\section{References}

1. Clermont G, Acker CG, Angus DC, Sirio CA, Pinsky MR, Johnson JP. Renal failure in the ICU: comparison of the impact of acute renal failure and endstage renal disease on ICU outcomes. Kidney Int. 2002;62:986-96.

2. Ishani A, Xue JL, Himmelfarb J, Eggers PW, Kimmel PL, Molitoris BA, et al. Acute kidney injury increases risk of ESRD among elderly. J Am Soc Nephrol JASN. 2009;20:223-8.

3. Coca SG, Yusuf B, Shlipak MG, Garg AX, Parikh CR. Long-term risk of mortality and other adverse outcomes after acute kidney injury: a systematic review and meta-analysis. Am J Kidney Dis Off J Natl Kidney Found. 2009;53:961-73. 
4. Chawla LS, Eggers PW, Star RA, Kimmel PL. Acute kidney injury and chronic kidney disease as interconnected syndromes. N Engl J Med. 2014;371:58-66.

5. Stafford-Smith M, Li Y-J, Mathew JP, Li Y-W, Ji Y, Phillips-Bute BG, et al. Genome-wide association study of acute kidney injury after coronary bypass graft surgery identifies susceptibility loci. Kidney Int. 2015;88:823-32.

6. Zhao B, Lu Q, Cheng Y, Belcher JM, Siew ED, Leaf DE, et al. A genome-wide association study to identify single-nucleotide polymorphisms for acute kidney injury. Am J Respir Crit Care Med. 2016;195:482-90.

7. Barasch J, Zager R, Bonventre JV. Acute kidney injury: a problem of definition. Lancet Lond Engl. 2017;389:779-81.

8. Copeland KT, Checkoway H, McMichael AJ, Holbrook RH. Bias due to misclassification in the estimation of relative risk. Am J Epidemiol. 1977;105: 488-95.

9. Siroux V, González JR, Bouzigon E, Curjuric I, Boudier A, Imboden M, et al. Genetic heterogeneity of asthma phenotypes identified by a clustering approach. Eur Respir J. 2014:43:439-52.

10. Bhatraju PK, Zelnick LR, Herting J, Katz R, Mikacenic C, Kosamo S, et al. Identification of acute kidney injury sub-phenotypes with differing molecular signatures and response to vasopressin therapy. Am J Respir Crit Care Med. 2018;199:863-72.

11. Russell JA, Walley KR, Singer J, Gordon AC, Hébert PC, Cooper DJ, et al. Vasopressin versus norepinephrine infusion in patients with septic shock. N Engl J Med. 2008;358:877-87.

12. Wu X, Guo R, Chen P, Wang Q, Cunningham PN. TNF induces caspasedependent inflammation in renal endothelial cells through a rho- and myosin light chain kinase-dependent mechanism. Am J Physiol Renal Physiol. 2009;297:F316-26.

13. Xu C, Chang A, Hack BK, Eadon MT, Alper SL, Cunningham PN. TNFmediated damage to glomerular endothelium is an important determinant of acute kidney injury in sepsis. Kidney Int. 2014;85:72-81.

14. Cunningham PN, Dyanov HM, Park P, Wang J, Newell KA, Quigg RJ. Acute renal failure in Endotoxemia is caused by TNF acting directly on TNF Receptor-1 in kidney. J Immunol. 2002;168:5817-23.

15. Han S, Lee S-J, Kim KE, Lee HS, Oh N, Park I, et al. Amelioration of sepsis by TIE2 activation-induced vascular protection. Sci Transl Med. 2016;8:335ra55.

16. Parikh SM. The Angiopoietin-Tie2 signaling Axis in systemic inflammation. J Am Soc Nephrol JASN. 2017;28:1973-82.

17. Wei $Y$, Tejera P, Wang Z, Zhang R, Chen F, Su L, et al. A missense genetic variant in LRRC16A/CARMIL1 improves acute respiratory distress syndrome survival by attenuating platelet count decline. Am J Respir Crit Care Med. 2017;195:1353-61.

18. Trinder M, Genga KR, Kong HJ, Blauw LL, Lo C, Li X, et al. Cholesteryl Ester transfer protein influences high-density lipoprotein levels and survival in Sepsis. Am J Respir Crit Care Med. 2019;199:854-62.

19. Reilly JP, Wang F, Jones TK, Palakshappa JA, Anderson BJ, Shashaty MGS, et al. Plasma angiopoietin-2 as a potential causal marker in sepsis-associated ARDS development: evidence from Mendelian randomization and mediation analysis. Intensive Care Med. 2018;44:1849-58.

20. Bhatraju PK, Mukherjee P, Robinson-Cohen C, O'Keefe GE, Frank AJ, Christie JD, et al. Acute kidney injury subphenotypes based on creatinine trajectory identifies patients at increased risk of death. Crit Care Lond Engl. 2016;20:372.

21. National Heart, Lung, and Blood Institute Acute Respiratory Distress Syndrome (ARDS) Clinical Trials Network, Matthay MA, Brower RG, Carson S, Douglas IS, Eisner M, et al. Randomized, placebo-controlled clinical trial of an aerosolized $\beta_{2}$-agonist for treatment of acute lung injury. Am J Respir Crit Care Med. 2011;184:561-8.

22. National Heart, Lung, and Blood Institute Acute Respiratory Distress Syndrome (Ards) Clinical Trials Network, Wheeler AP, Bernard GR, Thompson BT, Schoenfeld D, Wiedemann HP, et al. pulmonary-artery versus central venous catheter to guide treatment of acute lung injury. N Engl J Med 2006;354:2213-2224.

23. Rice TW, Wheeler AP, Thompson BT, de Boisblanc BP, Steingrub J, Rock P, et al. Enteral omega-3 fatty acid, gamma-linolenic acid, and antioxidant supplementation in acute lung injury. JAMA. 2011;306:1574-81.

24. Gong MN, Zhou W, Williams PL, Thompson BT, Pothier L, Christiani DC. Polymorphisms in the mannose binding lectin-2 gene and acute respiratory distress syndrome. Crit Care Med. 2007;35:48-56.

25. Liu KD, Glidden DV, Eisner MD, Parsons PE, Ware LB, Wheeler A, et al. Predictive and pathogenetic value of plasma biomarkers for acute kidney injury in patients with acute lung injury. Crit Care Med. 2007;35:2755-61.
26. Liu K-L, Lee K-T, Chang C-H, Chen Y-C, Lin S-M, Chu P-H. Elevated plasma thrombomodulin and angiopoietin-2 predict the development of acute kidney injury in patients with acute myocardial infarction. Crit Care Lond Engl. 2014;18:R100.

27. Siew ED, Matheny ME, Ikizler TA, Lewis JB, Miller RA, Waitman LR, et al. Commonly used surrogates for baseline renal function affect the classification and prognosis of acute kidney injury. Kidney Int. 2010;77:536-42.

28. Howie BN, Donnelly P, Marchini J. A flexible and accurate genotype imputation method for the next generation of genome-wide association studies. PLoS Genet. 2009:5:e1000529.

29. Marchini J, Howie B. Genotype imputation for genome-wide association studies. Nat Rev Genet. 2010;11:499-511.

30. Lee PH, Bergen SE, Perlis RH, Sullivan PF, Sklar P, Smoller JW, et al. Modifiers and subtype-specific analyses in whole-genome association studies: a likelihood framework. Hum Hered. 2011;72:10-20.

31. Bhatraju P, Hsu C, Mukherjee P, Glavan BJ, Burt A, Mikacenic C, et al. Associations between single nucleotide polymorphisms in the FAS pathway and acute kidney injury. Crit Care Lond Engl. 2015;19:368,

32. Yeboah J, Delaney JA, Nance R, McClelland RL, Polak JF, Sibley CT, et al. Mediation of cardiovascular risk factor effects through subclinical vascular disease: the multi-ethnic study of atherosclerosis. Arterioscler Thromb Vasc Biol. 2014;34:1778-83.

33. Kosuke Imai, Luke Keele, Teppei Yamamoto. Identification, Inference and Sensitivity Analysis for Causal Mediation Effects. JSTOR. 2010;25:51-71.

34. Skol AD, Scott $L$, Abecasis GR, Boehnke M. Joint analysis is more efficient than replication-based analysis for two-stage genome-wide association studies. Nat Genet. 2006:38:209-13.

35. Higgins SJ, Purcell LA, Silver KL, Tran V, Crowley V, Hawkes M, et al. Dysregulation of angiopoietin-1 plays a mechanistic role in the pathogenesis of cerebral malaria. Sci Transl Med. 2016;8:358ra128.

36. Pulvers JN, Journiac N, Arai $Y$, Nardelli J. MCPH1: a window into brain development and evolution. Front Cell Neurosci. 2015 [cited 2019 Mar 19];9. Available from: https://www.ncbi.nlm.nih.gov/pmc/articles/PMC4376118/.

37. Lieb W, Chen M-H, Larson MG, Safa R, Teumer A, Baumeister SE, et al. Genome-wide association study for endothelial growth factors. Circ Cardiovasc Genet. 2015;8:389-97.

38. Meyer NJ, Li M, Feng R, Bradfield J, Gallop R, Bellamy S, et al. ANGPT2 genetic variant is associated with trauma-associated acute lung injury and altered plasma angiopoietin-2 isoform ratio. Am J Respir Crit Care Med. 2011;183:1344-53.

39. Jongman RM, van Klarenbosch J, Molema G, Zijlstra JG, de Vries AJ, van Meurs M. Angiopoietin/Tie2 Dysbalance is associated with acute kidney injury after cardiac surgery assisted by cardiopulmonary bypass. PLoS One. 2015:10:e0136205.

40. Robinson-Cohen C, Katz R, Price BL, Harju-Baker S, Mikacenic C, Himmelfarb J, et al. Association of markers of endothelial dysregulation Ang1 and Ang2 with acute kidney injury in critically ill patients. Crit Care Lond Engl. 2016;20:207.

41. David S, Park J-K, van Meurs M, Zijlstra JG, Koenecke C, Schrimpf C, et al. Acute administration of recombinant Angiopoietin-1 ameliorates multipleorgan dysfunction syndrome and improves survival in murine sepsis. Cytokine. 2011:55:251-9.

42. Kümpers $P$, Hafer $C$, David $S$, Hecker H, Lukasz A, Fliser D, et al. Angiopoietin-2 in patients requiring renal replacement therapy in the ICU: relation to acute kidney injury, multiple organ dysfunction syndrome and outcome. Intensive Care Med. 2010;36:462-70.

43. Yuan HT, Khankin EV, Karumanchi SA, Parikh SM. Angiopoietin 2 is a partial agonist/antagonist of Tie2 signaling in the endothelium. Mol Cell Biol. 2009; 29:2011-22.

44. Kim DH, Jung YJ, Lee AS, Lee S, Kang KP, Lee TH, et al. COMP-angiopoietin1 decreases lipopolysaccharide-induced acute kidney injury. Kidney Int. 2009;76:1180-91.

45. Felcht M, Luck R, Schering A, Seidel P, Srivastava K, Hu J, et al. Angiopoietin2 differentially regulates angiogenesis through TIE2 and integrin signaling. J Clin Invest. 2012;122:1991-2005.

46. Fiedler $U$, Reiss $Y$, Scharpfenecker M, Grunow V, Koidl S, Thurston G, et al. Angiopoietin-2 sensitizes endothelial cells to TNF-alpha and has a crucial role in the induction of inflammation. Nat Med. 2006;12:235-9.

47. Higgins SJ, De Ceunynck K, Kellum JA, Chen X, Gu X, Chaudhry SA, et al. Tie2 protects the vasculature against thrombus formation in systemic inflammation. J Clin Invest. 2018;128:1471-84. 
48. Zacho J, Tybjaerg-Hansen A, Jensen JS, Grande P, Sillesen H, Nordestgaard BG. Genetically elevated C-reactive protein and ischemic vascular disease. N Engl J Med. 2008;359:1897-908.

49. Emerging Risk Factors Collaboration; Kaptoge S, Di Angelantonio E, Lowe G, Pepys MB, Thompson SG, Collins R, Danesh J. C-reactive protein concentration and risk of coronary heart disease, stroke, and mortality: an individual participant meta-analysis. Lancet. 2010;375(9709):132-40. https:// doi.org/10.1016/S0140-6736(09)61717-7.

\section{Publisher's Note}

Springer Nature remains neutral with regard to jurisdictional claims in published maps and institutional affiliations.

Ready to submit your research? Choose BMC and benefit from:

- fast, convenient online submission

- thorough peer review by experienced researchers in your field

- rapid publication on acceptance

- support for research data, including large and complex data types

- gold Open Access which fosters wider collaboration and increased citations

- maximum visibility for your research: over $100 \mathrm{M}$ website views per year

At $\mathrm{BMC}$, research is always in progress.

Learn more biomedcentral.com/submissions 